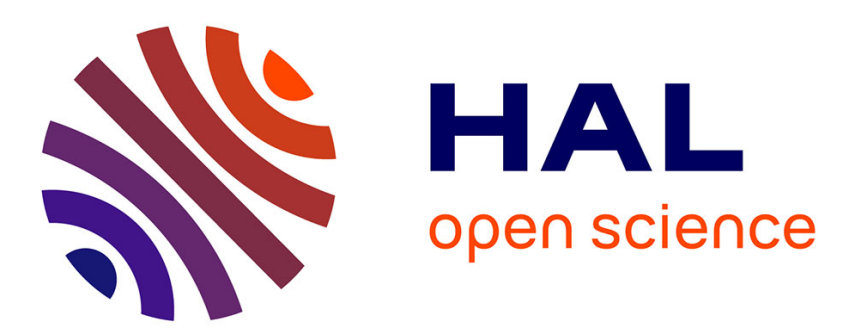

\title{
Loss of time-delay signature in the chaotic output of a semiconductor laser with optical feedback
}

\author{
D. Rontani, A. Locquet, M. Sciamanna, D. Citrin
}

\section{To cite this version:}

D. Rontani, A. Locquet, M. Sciamanna, D. Citrin. Loss of time-delay signature in the chaotic output of a semiconductor laser with optical feedback. Optics Letters, 2007, 32 (20), pp.2960-2962. 10.1364/OL.32.002960 . hal-03079798

\section{HAL Id: hal-03079798 \\ https://hal.science/hal-03079798}

Submitted on 17 Dec 2020

HAL is a multi-disciplinary open access archive for the deposit and dissemination of scientific research documents, whether they are published or not. The documents may come from teaching and research institutions in France or abroad, or from public or private research centers.
L'archive ouverte pluridisciplinaire HAL, est destinée au dépôt et à la diffusion de documents scientifiques de niveau recherche, publiés ou non, émanant des établissements d'enseignement et de recherche français ou étrangers, des laboratoires publics ou privés. 


\title{
Loss of time-delay signature in the chaotic output of a
}

\section{semiconductor laser with optical feedback}

\author{
D. Rontani ${ }^{1,2,3, *}$, A. Locquet ${ }^{2}$, M. Sciamanna ${ }^{1,2}$, and D. S. Citrin ${ }^{2,3}$ \\ ${ }^{1}$ Supélec, LMOPS CNRS UMR-7132, 2 Rue Edouard Belin, 57070 Metz, France \\ ${ }^{2}$ UMI 2958 Georgia Tech-CNRS, 2-3 Rue Marconi, 57070 Metz, France. \\ ${ }^{3}$ Georgia Institute of Technology, School of Electrical and Computer Engineering, Atlanta, \\ Georgia 30324-0250, USA \\ *Corresponding author: damien.rontani@supelec.fr
}

We investigate theoretically the possibility of retrieving the value of the time delay of a semiconductor laser with an external optical feedback from the analysis of its intensity time series. When the feedback rate is moderate and the injection current set such that the laser relaxation oscillation period is close to the delay, then the time-delay identification becomes extremely difficult, thus improving the security of chaos-based communications using external-cavity lasers. (C) 2007 Optical Society of America

OCIS codes: $140.1540,190.3100$

Chaotic cryptosystems exploit the noise-like appearance of a chaotic carrier to conceal an information-bearing message and the property of chaos synchronization [1] to decrypt 
this message at the receiving end. The security of a chaotic cryptosystem is twofold: first, it should be difficult to identify the emitter parameters and decipher the message by analyzing the transmitted signal, and second, even knowing the parameters, the physical realization of a synchronizing receiver should be arduous.

Semiconductor-based external-cavity lasers (ECLs) have proven to be a particularly interesting source of optical chaos [2]. Indeed, the delay introduced in the system can lead to the generation of high-dimensional chaos [3], which makes it computationally difficult to identify the system with traditional time-series-analysis techniques. Recently, some techniques tailored for delay systems have been developed $[4,5]$ which lead to computationally feasible system identification by working in a low-dimensional projection of the infinite-dimensional phase space. If the delay is unknown, however, it is impossible to perform such a projection and therefore to identify the system.

Previous works on time-delay identification in ECLs have shown that the delay was easily retrievable from the autocorrelation function of the laser light output $[6,7]$. The use of additional cavities has later been suggested [7] as a way to complicate the retrieval of information about the time delay; however, this makes practical implementation difficult.

In this Letter, we consider a single-mode semiconductor laser with a single external optical feedback. With this simple system, we show that for a careful choice of the operational parameters, it becomes extremely difficult to identify the time delay with the autocorrelationfunction and mutual-information techniques, both calculated from the intensity time series. The presence of two timescales, namely the relaxation-oscillation period $\tau_{R O}$ and the external- 
cavity round-trip time $\tau$, combined with appropriate values of the feedback rate $\gamma$, allows this masking. Our theoretical analysis is based on the Lang-Kobayashi (LK) rate-equation model [8],

$$
\begin{aligned}
\dot{E} & =\frac{1}{2}(1+i \alpha)\left(G-\frac{1}{\tau_{p}}\right) E+\gamma e^{i \omega_{0} \tau} E(t-\tau), \\
\dot{N} & =p J_{t h}-\frac{N}{\tau_{s}}-G|E|^{2},
\end{aligned}
$$

where $E$ is the slowly-varying complex electric field, $N$ is the average carrier density in the active region, $\alpha$ is the linewidth-enhancement factor, $G=G_{N}\left(N-N_{0}\right)\left(1-\varepsilon|E|^{2}\right)$ is the optical gain with $\varepsilon$ the saturation coefficient, $N_{0}$ is the carrier density at transparency, $\omega_{0}$ is the frequency of the solitary laser, $\gamma$ is the feedback rate, $\tau_{p}$ is the photon lifetime, $\tau_{s}$ is the carrier lifetime, $J_{t h}$ is the threshold current density, $p$ is the pumping factor, and $\tau$ is the delay. The relaxation oscillation period is an intrinsic damping time of the free running laser and is defined by $\tau_{R O}=\left(1 / \tau_{s} \tau_{p}(\mu-1)-\mu^{2} / 4 \tau_{s}^{2}\right)^{-1 / 2}$, with $\mu=G_{N} \tau_{s} \tau_{p}\left(p J_{t h}-N_{0} / \tau_{s}\right)$. We consider the following parameter values: $\alpha=5, \omega_{0} \tau=0$ $\tau_{p}=2 \mathrm{ps}, \tau_{s}=2 \mathrm{~ns}, G_{N}=7.5 \times 10^{-13} \mathrm{~m}^{3} \mathrm{~s}^{-1}, N_{0}=3 \times 10^{24} \mathrm{~m}^{-3}, \varepsilon=2.5 \times 10^{-23} \mathrm{~m}^{3}$, $J_{t h}=1.83 \times 10^{33} \mathrm{~m}^{-3} \mathrm{~s}^{-1}, \tau_{R O}=0.75 \mathrm{~ns}$. For a system modelled by a delay-differential equation, the information about the delay value can typically be retrieved using either the autocorrelation function or the mutual information [9]. The autocorrelation function is a measure of how well a signal matches a time-shifted version of itself and is defined by

$$
\rho_{I}(\theta)=\frac{\langle(I(t+\theta)-\langle I(t)\rangle)(I(t)-\langle I(t)\rangle)\rangle}{\left(\langle I(t)-\langle I(t)\rangle\rangle^{2}\langle I(t+\theta)-\langle I(t)\rangle\rangle^{2}\right)^{1 / 2}}
$$

where $I=|E|^{2}$ denotes the emitted laser intensity. 
The mutual information measures the information shared by two variables, namely, the intensities at times $t$ and $t+\theta$, averaged over $t$. It measures how much knowing one of these variables reduces our uncertainty about the other. We consider here the mutual information $\mathbf{I}(\theta)$ between $I(t)$ and $I(t-\theta)$ which is defined by

$$
\mathbf{I}(\theta)=\sum_{I(t), I(t-\theta)} p(I(t), I(t-\theta)) \log \frac{p(I(t), I(t-\theta))}{p(I(t)) p(I(t-\theta))}
$$

where $p(I(t), I(t-\theta))$ is the joint probability, and $p(I(t))$ and $p(I(t-\theta))$ are the marginal probability densities.

We now consider scenarios with various values of $\tau$ all leading to a chaotic behavior and we investigate circumstances under which the time delay is effectively concealed in the autocorrelation and mutual information. Usually, in delay systems, the signature of the time delay shows up in the autocorrelation function $\rho_{I}$ and the mutual information $\mathbf{I}$ because of the non-local time dependence induced by the delayed feedback term. Therefore, the information shared by $I(t)$ and $I(t-\tau)$ is intimately related to $\gamma$. Figures 1 and 2 analyze this influence. Figure 1 gives the shape of the autocorrelation function (left column) and the associated intensity time series (right column) with $\tau=5 \mathrm{~ns}$ for four different increasing values of $\gamma$. Figures 1.(d1) and 1.(c1) have the general shape described in the literature [7]: a central peak located at $\theta=0$ with a small temporal extension, and significant peaks at the delay and its multiples. These peaks are surrounded by small troughs at multiples of $\pm \tau_{R O} / 2$. The decrease of $\gamma$ induces a decrease of the peaks amplitude at the delay and its multiples; meanwhile, a significant trough at $\tau_{R O} / 2$ arises. For a smaller value of $\gamma$, we observe a decrease of the 
amplitude of the peaks at the delay and a significant peak appears at $\tau_{R O}$ (Fig.1.(b1)). For an even smaller value of the feedback rate (Fig. 1.(a1)), peaks appear at multiples of $\tau_{R O}$, revealing the importance of the relaxations oscillations in the dynamics. The presence of a second timescale due to the delayed feedback is revealed by the increase of the peaks value around $\tau$ and its multiples. In this latter case, delay identification is quite difficult due to the numerous satellite peaks present around $\tau$. Additionally, we expect that the delay will be significantly overestimated as the largest peak around $\tau$ corresponds to the multiple of $\tau_{R O}$ closest to the delay rather than to the delay itself.

Figure 2 analyzes the evolution with $\gamma$ of the location $\left(\tau_{W}\right)$ and the amplitude $\left(\rho_{W}\right)$ of the maximum autocorrelation peak in a time window around $\tau, W(\tau)=[3.5 \mathbf{n s}, 5.5 \mathbf{n s}]$. The time window is chosen to be sufficiently large to capture possible shifts of $\tau_{W}$, and sufficiently narrow to only measure the relevant peak.

The analysis of Fig. 2.(a), shows a continuous shift of $\tau_{W}$. It starts near a multiple of $\tau_{R O} / 2$ and then asymptotically tends to $\tau$. Figure 2.(b) shows a nonmonotonic evolution of $\rho_{W}$, which is consistent with the observations of Fig. 1(a1)-(d1) and unveils the existence of a minimum for a given feedback rate $\gamma$. These observations reveal the increasing reliability of $\tau_{W}$ with increasing values of $\gamma$, because the choice of the peak in the autocorrelation becomes more obvious and simultaneously the delay overestimation decreases. Consequently, the system becomes less and less secure, regarding system identification. It is also known that increasing $\gamma$ leads to an increase of the Kaplan-Yorke dimension in a LK system [3]. Our result shows 
that the high dimensionality of the chaos is not a sufficient condition for security, as delay identification becomes easier when the feedback rate, and thus the dimension, is increased.

From the first two figures, the relative importance of the two time scales in the autocorrelation function, controlled by the value of $\gamma$, has proven to be of key importance for the delay retrieval. The signatures of the delay and of the relaxation-oscillations period are always present in the autocorrelation function as illustrated in Fig. 1. At a moderate feedback rate (Fig. 1.(a)), there are numerous large peaks in the vicinity of $\theta=0$, corresponding to small multiples of $\tau_{R O} / 2$, and similarly in the vicinity of $\tau$, higher multiples of $\tau_{R O} / 2$ are present. The presence of peaks at multiples of $\tau_{R O}$ perturbs the delay identification but the significant separation between the values of $\tau$ and $\tau_{R O}$ still allows a relatively clear identification of the delay value, as we observe a clear increase around the value of $\tau$ of peaks at high-order multiples of $\tau_{R O}$. We find that reducing the separation between $\tau$ and $\tau_{R O}$ makes the identification problematic. Figure 3 illustrates such a case using the autocorrelation function (left column) and mutual information (right column) considering $\tau=1 \mathrm{~ns}$. The ECL behavior is chaotic for the four increasing values of $\gamma$ considered in Fig. 3. Figures 3.(d1) and 3.(d2) confirm the results obtained in Fig. 1: sharp peaks are located at the delay and its multiples. As $\gamma$ decreases from 15 to $10 \mathrm{GHz}$, the peak close to the delay value in both $\rho_{I}$ and $\mathbf{I}$ decreases (Figs. 3.(c1)-(c2)). A further decrease of $\gamma$ to $5 \mathrm{GHz}$ reveals very interesting plots of the autocorrelation function and the mutual information. In Figs. 3.(b1) and 3.(b2), we do not observe any clearly identifiable peaks around the delay value. The only significant peaks or troughs being located around $\tau_{R O} / 2$, an eavesdropper would 
have an exceedingly difficult time to identify the delay. Finally Figs. 3.(a1) and (a2), which correspond to a feedback rate of $2.5 \mathrm{GHz}$, also show a case in which it is seems impossible to identify the delay as only a minor trough or peak is visible around the delay value and as an eavesdropper would have no indication that these correspond to the correct delay value.

A systematic numerical study shows that the delay is well concealed when $\tau$ belongs to the interval $\left[\tau_{R O}+0.15 \mathbf{n s}, \tau_{R O}+0.35 \mathbf{n s}\right]$.

In conclusion, we have demonstrated, using autocorrelation and mutual-information techniques, the key role of the feedback rate $\gamma$ and of the separation between the time delay $\tau$ and the period of the relaxation oscillation $\tau_{R O}$ on the retrieval of the time delay in an ECL. Two scenarios have been identified. First, for low feedback rates and significant separation between $\tau$ and $\tau_{R O}$, the presence of peaks or troughs at multiples of $\tau_{R O} / 2$ makes the identification difficult. Second, when the feedback rate is low and the values of $\tau$ and $\tau_{R O}$ are close, delay identification is very difficult because no significant peak appears close to the delay value. These two situations can prevent an eavesdropper to access the true measure of the time delay.

The authors acknowledge the support of Région Lorraine and fondation Supélec. D. S. Citrin was supported in part by the NSF, by grant ECCS 0523923.

\section{References}

1. L.M. Pecora and T.L Carroll, Phys. Rev. Lett. 64, 821-824 (1990). 
2. A. Argyris, D. Syvridis, L. Larger, V. Annovazzi-Lodi, P. Colet, I. Fischer, J. GarciaOjalvo, C.R Mirasso, L. Pesquera, and K.A Shore, Nature 437, 343-346 (2005).

3. R. Vicente, J. Daudén, P. Colet, and R. Toral, IEEE J. Quantum Electron. 41, 4, 541 $-548(2005)$.

4. M.J. Bünner, M. Popp, Th. Meyer, A. Kittel, and J. Parisi, Phys. Rev. E 54 4, 3082-3085 (1996).

5. R. Hegger, M.J. Bünner, H. Kantz, and A. Giaquinta, Phys. Rev. Lett. 81, 3, 558-561 (1998).

6. M.J. Bünner, A. Kittel, J. Parisi, I. Fischer, and W. Elsaßer, Europhys. Lett. 42, 353-358 (1998).

7. M.W Lee, P. Rees, K.A. Shore, S. Ortin, L. Pesquera, and A. Valle, IEE Proc. Optoelectron. 152, 97-102 (2005).

8. R. Lang and K. Kobayashi, IEEE J. Quantum Electron. 16, 347 (1980).

9. V.S Udaltsov, L. Larger, J.P Goedgebuer, A. Locquet, and D.S. Citrin, J. Opt. Technol. 72, 5, 373-377 (2005). 


\section{References}

1. L.M. Pecora and T.L Carroll, "Synchronization in chaotic systems", Phys. Rev. Lett. 64, 821-824 (1990).

2. A. Argyris, D. Syvridis, L. Larger, V. Annovazzi-Lodi, P. Colet, I. Fischer, J. GarciaOjalvo, C.R Mirasso, L. Pesquera, and K.A Shore, "Chaos-based communications at high bit rates using commercial fibre-optic links", Nature 437, 343-346 (2005).

3. R. Vicente, J. Daudén, P. Colet, and R. Toral, "Analysis and characterization of the hyperchaos generated by a semiconductor laser subject to a delayed feedback loop", IEEE J. Quantum Electron. 41, 4,541 - 548 (2005).

4. M.J. Bünner, M. Popp, Th. Meyer, A. Kittel, and J.Parisi, "Tool to recover scalar timedelay systems from experimental time series", Phys. Rev. E 54 4, 3082-3085 (1996).

5. R. Hegger, M.J. Bünner, H. Kantz, and A. Giaquinta, "Identifying and Modeling Delay Feedback Systems", Phys. Rev. Lett. 81, 3, 558-561 (1998).

6. M.J. Bünner, A. Kittel, J. Parisi, I. Fischer, and W. Elsaßer, "Estimation of delay times from a delayed optical feedback laser experiment", Europhys. Lett. 42, 353-358 (1998).

7. M.W Lee, P. Rees, K.A. Shore, S. Ortin, L. Pesquera, and A. Valle, "Dynamical characterisation of laser diode subject to double optical feedback for chaotic optical communications", IEE Proc. Optoelectron. 152, 97-102 (2005).

8. R. Lang and K. Kobayashi, "External optical feedback effects on semiconductor injection laser properties", IEEE J. Quantum Electron. 16, 347-355 (1980). 
9. V.S Udaltsov, L. Larger, J.P Goedgebuer, A. Locquet, and D.S. Citrin, "Security of chaos-based communication system ruled by Delay Differential Equation. Recovery of time-delay", J. Opt. Technol. 72, 5, 373-377 (2005).

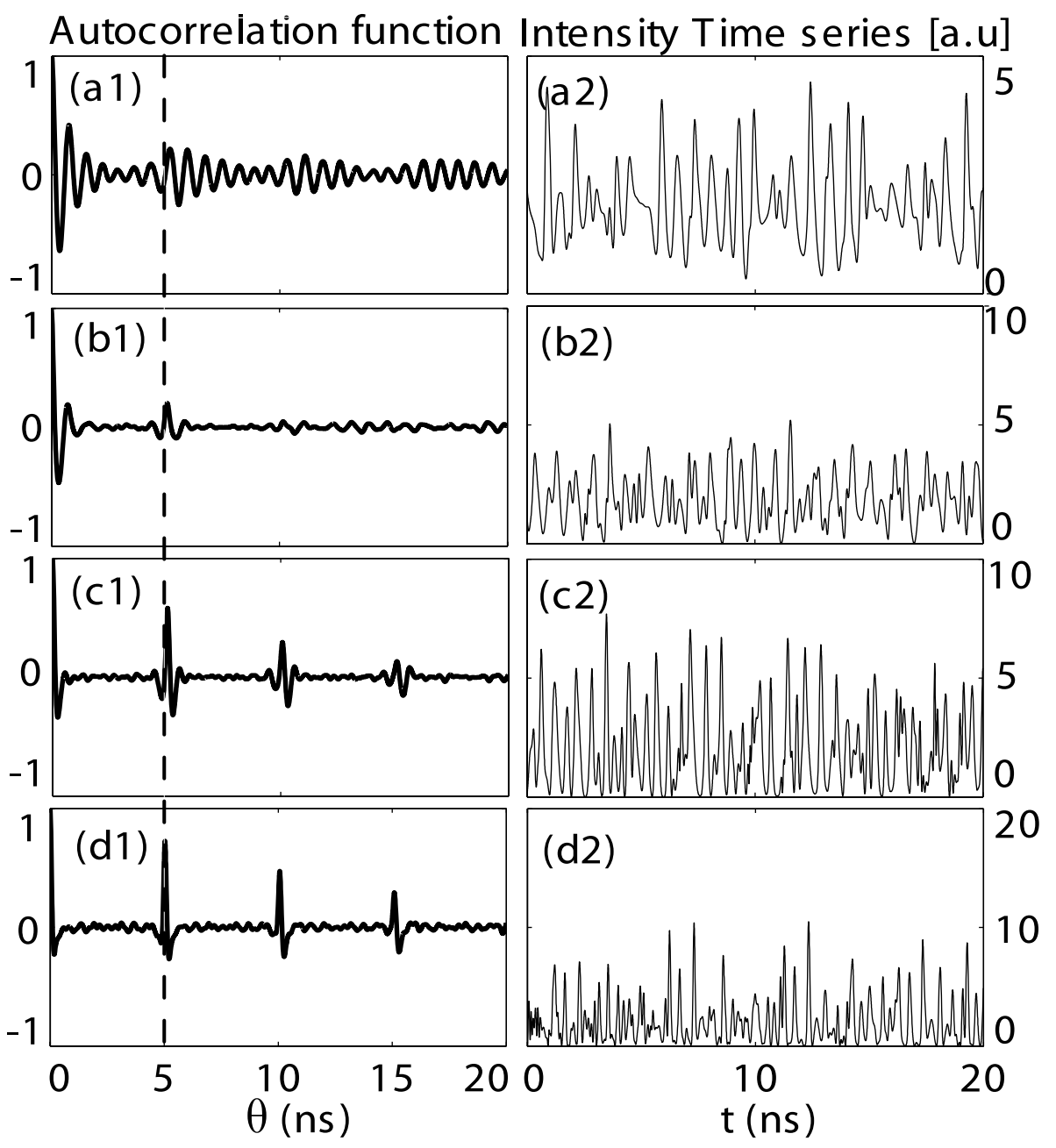

Fig. 1. Autocorrelation function and associated time series $\tau=5 \mathrm{~ns}, \tau_{R O}=0.75 \mathrm{~ns}$, and (a1)(a2) $\gamma=2.5 \mathrm{GHz},(\mathrm{b} 1)-(\mathrm{b} 2) \gamma=5 \mathrm{GHz},(\mathrm{c} 1)-(\mathrm{c} 2) \gamma=10 \mathrm{GHz}$ and $(\mathrm{d} 1)-(\mathrm{d} 2) \gamma=15 \mathrm{GHz}$. The vertical dashed line indicates $\tau$. 


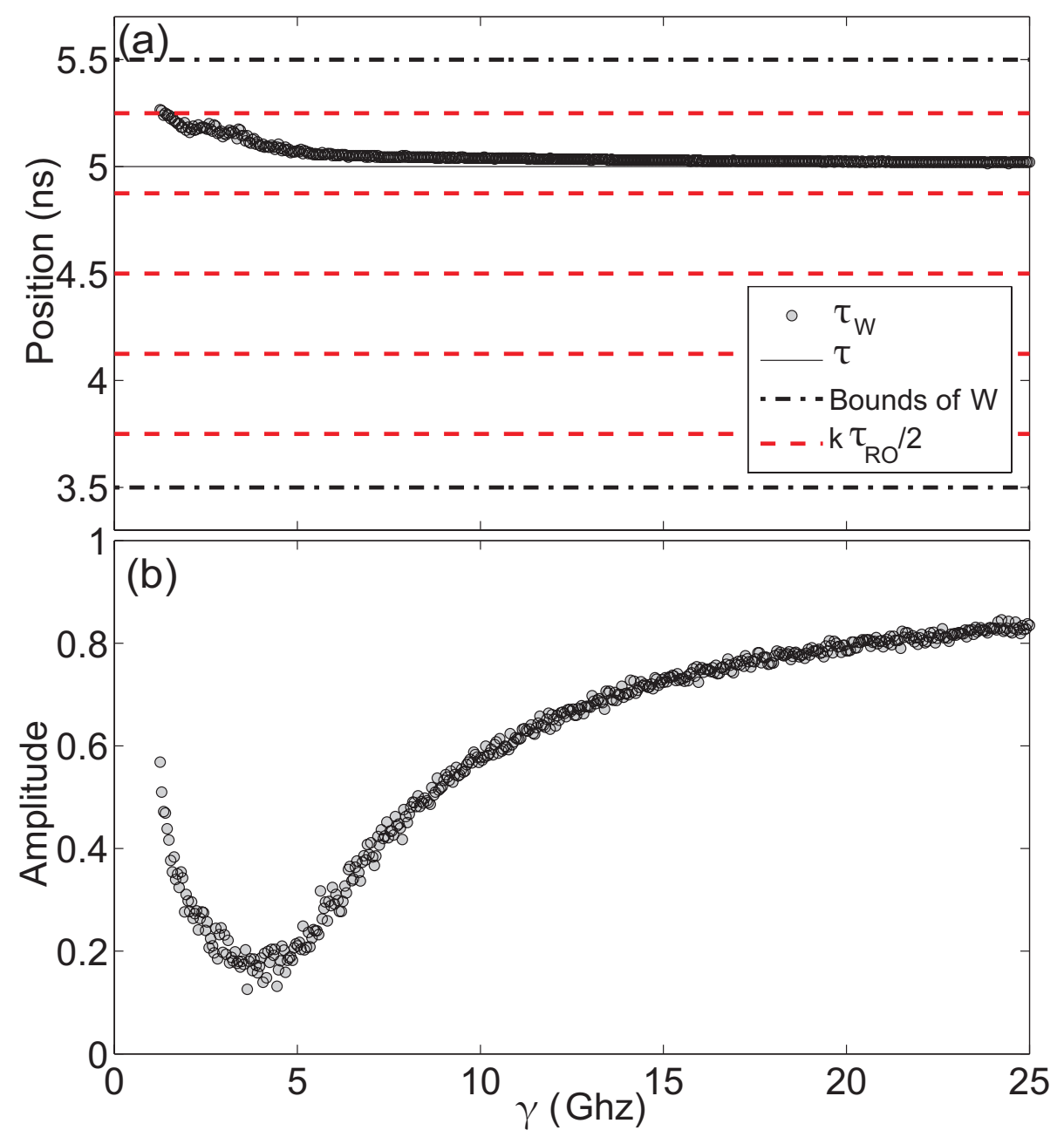

Fig. 2. Evolution of the position and amplitude of the maximum autocorrelation peak for $\tau=5 \mathrm{~ns}, \tau_{R O}=0.75 \mathrm{~ns}$, in a window $W(\tau)$, as a function of $\gamma$. Dashed lines show multiples of $\tau_{R O} / 2$. Dotted-dashed lines show the boundaries of $W(\tau)$. The solid line indicates $\tau$. 


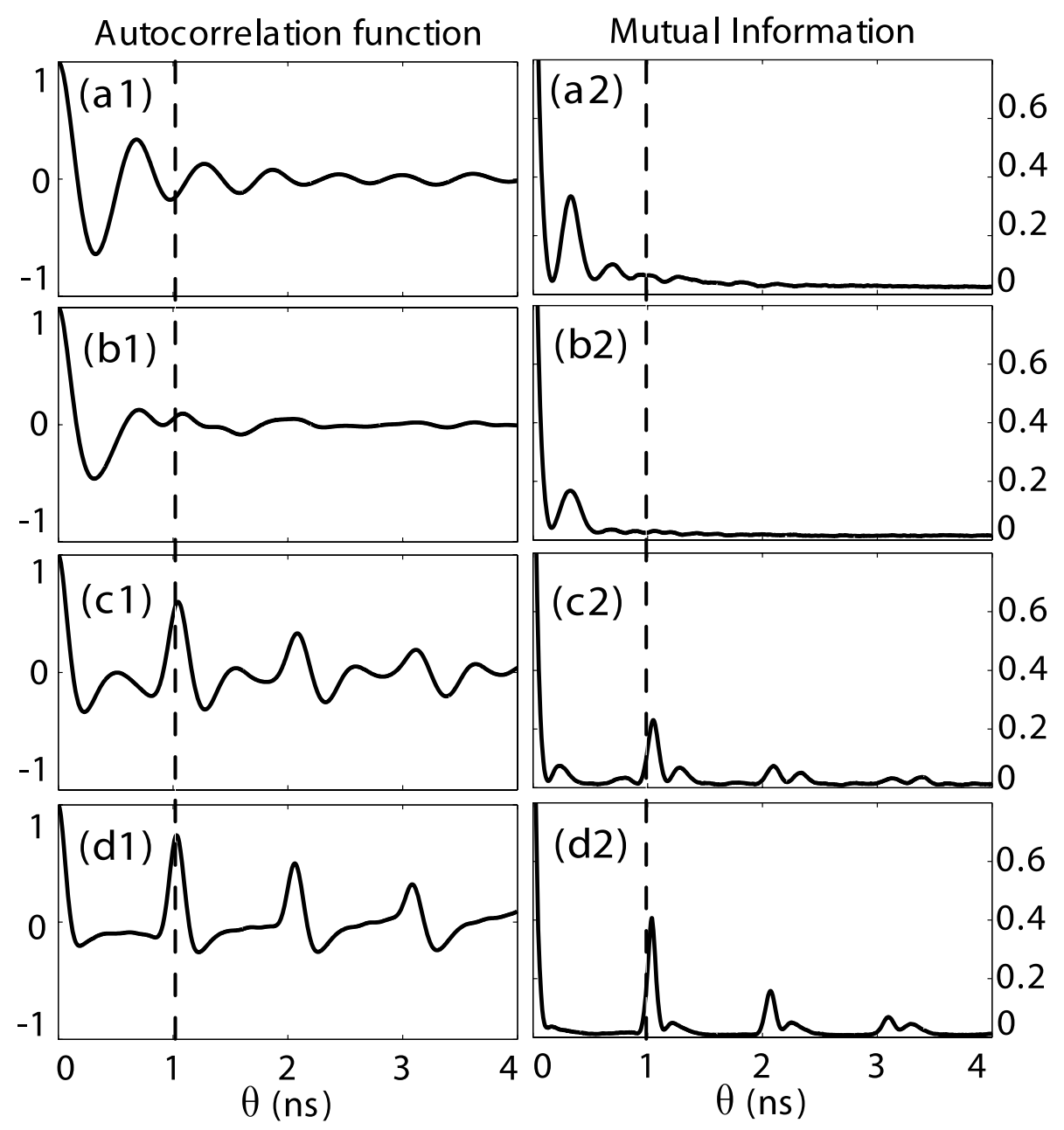

Fig. 3. Autocorrelation function and mutual information for $\tau=1 \mathrm{~ns}$ and $\tau_{R O}=0.75 \mathrm{~ns}$. (a1)(a2) $\gamma=2.5 \mathrm{GHz},(\mathrm{b} 1)-(\mathrm{b} 2) \gamma=5 \mathrm{GHz},(\mathrm{c} 1)-(\mathrm{c} 2) \gamma=10 \mathrm{GHz}$ and $(\mathrm{d} 1)-(\mathrm{d} 2) \gamma=15 \mathrm{GHz}$ The vertical dashed line indicates $\tau$. 